to demand a guarantee that the milk supplied is free from tuberculosis as shown by the tuberculin test.

Tuberculosis should also, according to Dr. Niven, be made a compulsorily notifiable disease, and the persons affected must be followed up. The lodging houses must be inspected, and of course disinfected, and then on the important paragraph, "It is needless to enter on the subject of isolation of cases of tuberculosis at present. When we have once obtained notification of therefrom the administrative measures which arise All may be left to develop.'

All meat must be inspected, and inspectors must be to milk, the tubed to recognise tuberculosis. In regard ployed; or, in its abulin test to the cows must be emmilk from each for every pailful of of tubercle bacillim should be tested for the presence cally examined by a veterinary should be systematiThis means the establinary surgeon in every district. Health Service." The medist of a Veterinary Public be empowered to order medical officer of health should to yield tuberculous milk slaughter of any cow shown to oblige landlords to and powers should be obtained accordance with the model regulation buildings in by the Local Government regulations recommended

This is but a rough soard.

made, from which it match of some of the proposals subject, and may be judged how large is the when dealt how far-reaching are its ramifications by Dr. Niven.

\section{CLINICAL SOCIETY.}

Dr. W. Pasteur igave an account of an epidemic of infantile paralysis among children of the same family. Seven children were affected within the space of ten days. In all there were febrile manifestations with marked headache, which in three cases were followed by paralysis and in two by tremors, the latter being of short duration. One case was a typical one of infantile rently of cerebral was of hemiplegic type and appaaddition to a paral origin, while a third presented, in at the outset. great interest. It was, he thought, a matter of very cases. It seemed clear that was the nature of these were all casually connected whatever their origin they that they were connected with the primary fever, and infective process. eruptive fevers, nor was it diphtheris not one of the influen the parents had restrict, besides which the health of selves had shown remained good, and the children themthen, of the fact no symptoms of catarrh. In view, were the result of that, whatever these cases were, they of them presented same infective process, while one paralysis, it seemed the usual features of infantile change was probabl to Dr. Pasteur that the morbid occurred in probably of the same nature as that which different situations, in poliomyelitis, but occupying above the level of rant forms of infant pons. They were, in fact, aberinterest was, firstly, that paralysis, and the point of order could arise frat such varied forms of nerve disfantile paralysis was the same cause, showing that in- some had imagined, and, secondly, that infantile paralysis was a specific infective malady.

The President (Dr. Buzzard) said that the paper read by Dr. Pasteur was of much interest as being, he thought, the first occasion on which the infective character of the disease causing infantile paralysis had been brought before an English medical society. The cases related by Dr. Pasteur and those of Dr. Medin were mutually confirmatory of each other. He felt very sure that infantile paralysis arose from some toxic influence, and that this was not limited to the anterior cornua of the spinal cord. In Medin's cases 27 of them were typical of anterior poliomyelitis, showing that there could be no doubt as to the nature of the disease, yet in the rest of the cases the morbid changes were distributed over different portions of the cerebro-spinal system; and it seemed clear that some toxic agent, to which we could not yet give a name, was associated with a certain pyrexial disease, and might in some cases fall on the anterior cornua, while in others its effects might be distributed over different parts of the cerebral and other centres. He related a case in which a little boy was attacked with infantile paralysis and it was found that at the same time that he had the febrile stage a little sister had a febrile attack of the same nature, but not ending in paralysis. Dr. Buzzard said he was in the habit of directing his clerks to inquire not only as to antecedent illness among relatives, but also as to coincident illness among other children in the house or neighbourhood. Nothing could be more sad than the condition in which children were left for their lives by this disease, and it was of great importance that those who had the opportunity of seeing such cases at the very beginning should investigate the blood bacteriologically at the earliest possible moment, in the hope that some bacillary cause of the malady might be discovered, and that an anti-toxin might be found, by the immediate use of which some of these disastrous consequences might be prevented,

\section{ROYAL MEDICAL AND CHIRURGICAL SOCIETY.}

At the last meeting, Dr. Duggan read a paper on "The "Parasite of the Malarial Fevers at Sierra Leone." During two years' residence he had examined blood from the finger in nearly 400 cases. After describing the symptoms met with, he said that in all cases he found the parasite in blood from the finger. He then described the appearances observed, which were similar to those described by Marchiafava as being found in the summer-autumn fevers of Rome. Morphologically, the author of the paper was not able to detect any difference between Marchiafava's parasite and that of Sierra Leone. In the discussion, Dr. Thin said that the fevers on the West Coast of Africa were extremely fatal as compared with those in the South of Europe. This, he thought, was due to a plurality of parasites. As illustrating the dangers to Europeans, he mentioned an instance in which four out of five artisans died from fever almost immediately after arrival in the country; one of them being attacked on the second day (which showed an unusually short period of incubation) and dying on the fourth. It was remark able what a protective influence the regular administration of quinine had in regard to this disease. Dr. Manson insisted on the importance of microscopic examination of the blood in such cases fo: purposes c $f$ diagnosis, for many cases which were now known to be cases of severe malaria would formerly have been returned as cases of sunstroke. 